\title{
Direct 3D observation and unravelling of electroconvection phenomena during concentration polarization at ion-exchange membranes
}

\author{
Felix Stockmeier ${ }^{\mathrm{a}, \mathrm{b}}$, Michael Schatz ${ }^{\mathrm{a}, \mathrm{b}}$, Malte Habermann ${ }^{\mathrm{a}, \mathrm{b}}$, John \\ Linkhorst $^{\mathrm{a}}$, Ali Mani ${ }^{\mathrm{c}}$, Matthias Wessling ${ }^{\mathrm{a}, \mathrm{b}, *}$ \\ ${ }^{a}$ Chemical Process Engineering AVT.CVT, RWTH Aachen University, Forckenbeckstraße \\ 51, Aachen, 52074, Germany \\ ${ }^{b} D W I$ - Leibniz-Institute for Interactive Materials, Forckenbeckstraße \\ 50, Aachen, 52074, Germany \\ ${ }^{c}$ Department of Mechanical Engineering, Stanford \\ University, Stanford, 94305, California, USA
}

\begin{abstract}
A decade ago, two-dimensional microscopic flow visualization proved the theoretically predicted existence of electroconvection roles as well as their decisive role in destabilizing the concentration polarization layer at ion-selective fluid/membrane interfaces. Electroconvection induces chaotic flow vortices injecting volume having bulk concentration into the ion-depleted diffusion layer at the interface. Experimental quantification of these important flow patterns have so far only been carried out in 2D. Numerical direct simulations suggest 3D features, yet experimental proof is lacking. 3D simulations are also limited in covering extended spacial and temporal scales.

This study presents a new comprehensive experimental method for the time-resolved recording of the 3D electroconvective velocity field near a cationexchange membrane. For the first time, the spatio-temporal velocity field can be visualized in 3D at multiples of the overlimiting current density. In contrast to today's simulations, these experiments cover length and time scales typical for actual electrodialytic membrane processes.

We visualize coherent vortex structures and reveal the changes in the velocity field and its statistics during the transition from vortex rolls to vortex
\end{abstract}

${ }^{*}$ Corresponding author:

Email address: manuscripts.cvt@avt.rwth-aachen.de 
rings with increasing current density. The transition is characterized by changes in the rotation direction, mean square velocity, and temporal energy spectrum with only little influence on the spatial spectrum. These findings indicate a more significant impact of EC's structural change on the mean square velocities and temporal spectra than on the spatial spectra. This knowledge is a prerequisite for engineering ion-selective surfaces that will enable the operation of electrically driven processes beyond the diffusionlimited Nernst regime.

Keywords: Ion transport, Electroconvection, 3D PTV, Velocity field, Electrodialysis

\section{Introduction}

Ion-selective membranes are frequently used in industrial processes for water purification such as electrodialysis (ED) $[1,2]$ and flow-electrode capacitive deionization (FCDI) $[3,4,5]$, or in systems for energy storage like redox-flow battery systems $[6,7]$. In these processes, an electric potential between two electrodes acts as the driving force for ion transport through membranes in contact with an electrolyte. For small currents, the current density increases linearly with the applied potential. However, the transport stagnates at a limiting current density $i_{\text {lim }}$ when concentration polarization (CP) leads to depletion of the Nernst diffusion layer close to the membrane, see Fig. 1 a) [8]. This limitation is visible as a plateau region in a current density over potential plot in Fig. 1 b).

When increasing the current density beyond $i_{\text {lim }}$, overlimiting currents emerge having their origin in a variety of superimposed phenomena, one of them being electroconvection (EC). Electroconvection can be observed as vortices, which convectively mix the boundary layer supplying the depleted layer with the ion-rich bulk solution, see Fig. 1 a). The increased ion concentration at the membrane allows for an overlimiting current beyond the diffusion limit, see Fig. 1 b) [9].

Until now, quantitative studies on the hydrodynamics of EC were limited to computationally demanding $2 \mathrm{D}$ and $3 \mathrm{D}$ direct numerical simulations (DNSs) which unfortunately only offer limited length and time scales [10, 11, 12, 13, 14]: experimental quantification of velocity profiles could only be done using $2 \mathrm{D}$ experiments $[15,16,17]$. In fact, understanding and controlling the hydrodynamics of EC is expected to facilitate mass transport 
observed as overlimiting currents. Enabling higher current densities results in lower membrane area required to achieve a desired desalination degree: this results in a desired investment costs reduction. It is therefore obvious that understanding the details of the overlimiting transport, in particular $\mathrm{EC}$, is a prerequisite for process optimization of an electrical-field driven membrane process. Also, understanding electroconvection's intricate details comprehensively is important to tailor membrane surfaces such that overlimiting can be actually utilized in practice.

\section{Background}

In a milestone paper of Rubinstein [18], the authors predicted that the onset of electroconvection can be triggered at lower voltages if the surface of the membrane would be "wavy". This prediction has spurred research in understanding the phenomena itself, but also into the synthesis of new membrane surface topologies to avoid any potential drop in the plateau region prior to the overlimiting currents. With respect to experimental strategies to initiate early electroconvection, membrane modification methods developed by the Kuban research community have proven to be effective [19, 20, 21]. As early as 2007, Balster reported that line undulations on the membrane surface normal to the flow direction, having distances in the range of approximately 50-200\% of the boundary-layer thickness, lead to an earlier onset of the overlimiting current [22]. Also 2D micropatterns of nanometer-thick lateral polyelectrolyte patches induce electroconvection, i.e. macrosopic electroosmotic chaotic fluid instabilities [23]. Also Roghmans et al. described that inkjet printed microgel patterns induce electroconvection [24]. Yet the work hypothesizes that the surface charge of the patterns may even determine the direction of vortices rendering them either effective or less effective for destabilizing the diffusion boundary layer and reducing the length of the plateau. Clearly, more experimental visualization methods are highly desired to elucidate and quantify the spatio-temporal fluid velocities during electroconvection at the membrane/fluid interface.

Historically, our 2D work on proving the existence of electroconvection through visualization with a particle imaging technique [25] confirmed the predicted vortex-like features at the membrane/liquid interface with growing vortices at increasing current densities. Subsequently, De Valenca et al. quantified the $2 \mathrm{D}$ velocity field of $\mathrm{EC}$ in the cross-section of the CP layer $[15,16]$. They correlated the vortex size and fluid flow velocity with the cur- 
a)
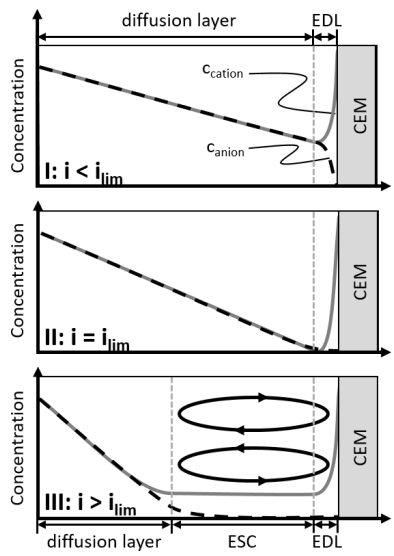

b)

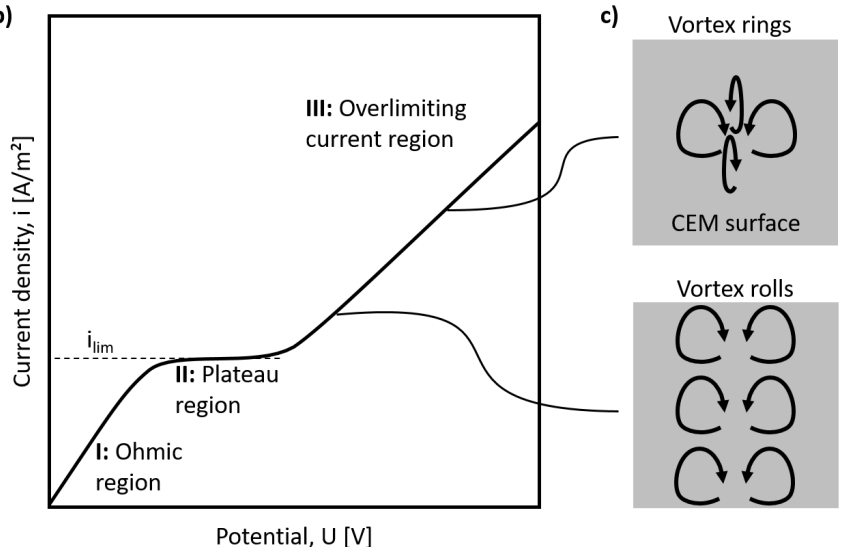

Figure 1: Stages of electroconvection. a) Concentration profiles of anions and cations in front of the membrane for three different regimes (I: $i<i_{\text {lim }}$, II: $i=i_{\text {lim }}$, and III: $i>i_{\text {lim }}$ ). b) Sketch of the current versus voltage curve for an electrically driven membrane process. The three regimes in a) appear as the Ohmic, plateau, and overlimiting regions. c) Sketches of the different vortex structures developing in the overlimiting regime. First, EC appears as vortex rolls that order in a $2 \mathrm{D}$ fashion. With increasing $i / i_{\text {lim }}$, the linear structure of vortex rolls breaks up into $3 \mathrm{D}$ vortex rings.

rent density through the system. This approach was recently advanced by Warren et al. [17] who correlated the effect of the applied potentials to the 2D velocity field of EC. However, their proposed electrochemical chip had an aspect ratio non-existent in common membrane modules. Other studies also analyzed EC by optically recording tracer substances in $2 \mathrm{D}$ but without quantification of velocities [25, 26, 27, 28, 29, 30, 31]. Recently, Kang et al. visualized the structural transition of EC in the overlimiting current region with and without flow parallel to a membrane surface with confocal microscopy in 3D [32]. This transition from $2 \mathrm{D}$ vortex rolls towards 3D vortex rings, see Fig. 1 c), was predicted by Demekhin et al. in the first 3D DNSs of EC [11]. Although qualitatively accurate, such confocal microscopy methods do not supply information on the 3D EC velocity field and its energy transfer.

The chaotic 3D velocity field of EC is up to now only accessible via intensive simulations simultaneously solving the Navier-Stokes, Nernst-Planck, and Poisson-Boltzmann equations [9]. In such a simulation, Druzgalski et al. analyzed and compared the statistics of chaotic EC form 2D to 3D results in an attempt to derive energy spectra needed for the development of a 
statistically averaged reduced-order model $[10,12]$. Such a statistical averaged model would enable low-cost simulations in the overlimiting regime for process optimization [9]. Experimentally, a simple reduced-order parameter approach has been suggested based on fitting the overlimiting currents utilizing a Schmidt number [33]. However, this approach has not been further explored until today. Furthermore, Druzgalski et al. also found qualitative and quantitative differences in their 2D and 3D simulations, emphasizing the need for an new methodology quantifying the 3D hydrodynamics. The required fine resolution in space and time limited their simulations to small geometries and short time spans only. Therefore, the ability to extrapolate their conclusions to the length and time scales of practically relevant devices remains unclear until experimental results of the 3D velocity field are available.

To overcome this lack of experimental data, we developed and report an electrochemical cell allowing the optical recording of the 3D EC velocity field close to a cation-exchange membrane using micro particle tracking velocimetry $(\mu \mathrm{PTV})$. With this setup, one can now make the important step from $2 \mathrm{D}$ to 3D velocity field quantification: at practically relevant length and time scales over a range of overlimiting current densities. We use the velocity data to analyze the structural change of EC from vortex rolls to vortex rings in terms of vortex rotation direction. Additionally, we evaluate the velocity field statistics and the fluctuating velocity component. This proposed methodology allows to unravel details of EC hydrodynamics experimentally which in future can be compared to simulated velocity statistics when the reported length and time-scales will be accessible through high performance computing efforts.

\section{Experimental Methods}

\subsection{Electrochemical cell}

We designed the experimental cell shown in Fig. 2 which is suitable for electrochemical experiments with simultaneous recording of the evolving 3D velocity field using microparticle tracking velocimetry ( $\mu \mathrm{PTV})$. The cell is based on the publication of Linz et al. [34] and consists of a Nafion N117 (Chemours, Wilmington, Delaware, USA) cation-exchange membrane sealed between two housing parts. The transparency of the membrane allows for imaging of buoyancy stable EC through the membrane [35, 36]. Two copper 


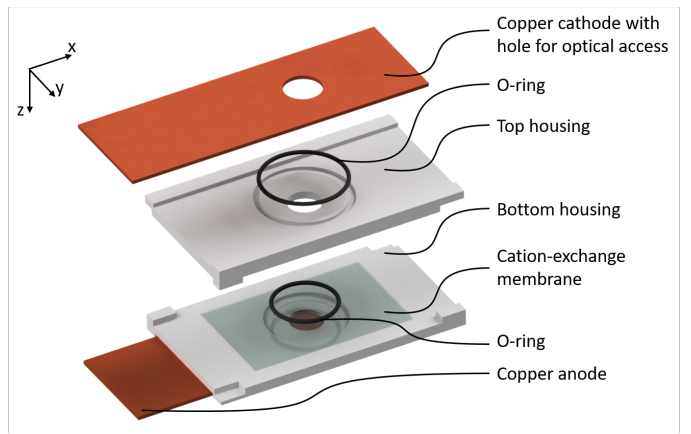

Figure 2: Cell design for electrochemical experiments with simultaneous $\mu$ PTV recording. The electrochemical cell consists of of two 3D printed housing parts which are sealed with O-rings against two copper electrodes and a Nafion N117 membrane. The cathode has a circular hole with a diameter of $9 \mathrm{~mm}$ to allow optical access. The hole is sealed with a microscope glass slide, which is not shown in the rendering, on top of the electrode.

plates $(25 \mathrm{~mm} \times 75 \mathrm{~mm} \times 0.5 \mathrm{~mm})$ seal the top and the bottom of the chip and act as electrodes in each half cell. The cathode has a circular hole $(\mathrm{d}=$ $9 \mathrm{~mm}$ ) and is glued to a glass slide to enable optical access. This setup forms electrolyte chambers above and below the membrane, which are filled with $1 \mathrm{mM}$ copper sulfate $\left(\mathrm{CuSO}_{4}\right)$ as the electrolyte. The bottom chamber has a height of $0.8 \mathrm{~mm}$, matching the maximum focal depth, and a diameter of $8 \mathrm{~mm}$, resulting in an aspect ratio of 10 . Such a large aspect ratio is desired to prevent the confinement of the EC vortices [37, 38].

A cell comprising two copper electrodes, a cation-exchange membrane, and $\mathrm{CuSO}_{4}$ in aqueous solution as electrolyte is a simple and well studied electrochemical system for evoking $\mathrm{EC}[15,39]$. In this system, $\mathrm{CuSO}_{4}$ dissociates to $\mathrm{Cu}^{2+}$ and $\mathrm{SO}_{4}{ }^{2-}$ and both electrodes favor copper deposition and dissolution as faradaic reactions [40].

During operation, copper is dissolved at the anode, transported through the membrane, and deposited on the cathode. Additionally, this reaction system limits gas evolution at the electrodes and the membrane to a minimum, which ensures stable reaction conditions and clear optical access without gas bubbles.

The copper ions moving towards the cathode result in an ion flux i per membrane area also describes as the current density. The limiting current density of the process, described in Fig. 1, can be calculated by [16]: 


$$
i_{\lim }=\frac{c_{B}}{\delta} \cdot \frac{F \cdot z}{t_{M}-t_{E}}
$$

\subsection{Micro particle image velocimetry}

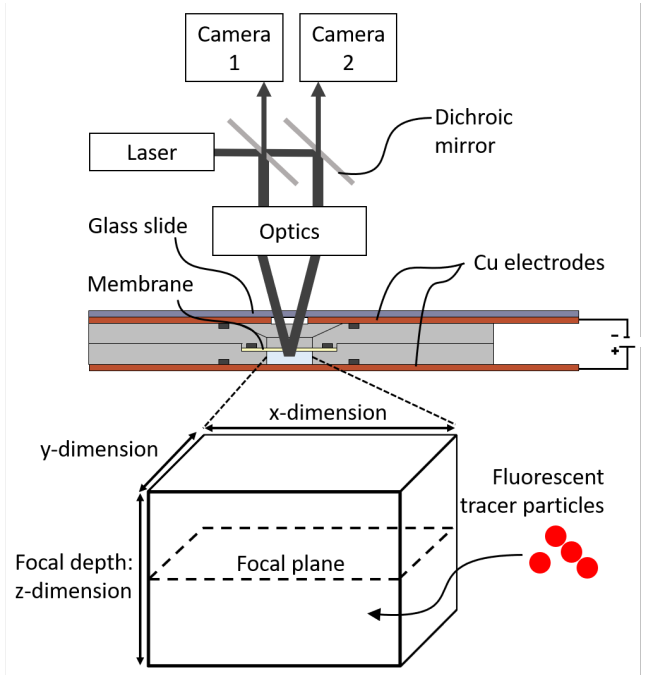
of the anode chamber [15].

Figure 3: Schematic illustration of the setup for stereo micro particle image velocimetry. The setup for stereo micro particle image velocimetry consists of a laser that illuminates tracer particles in a sample volume through a stereo microscope which splits the laser beam in two separate beams. The fluorescence response returns through the microscope and is recorded by two slightly angled high-speed cameras. Returning laser light is blocked by a high-pass filter. This technique allows the recording of tracer particles in a $3 \mathrm{D}$ volume.

According to de Valenca [16], the bulk concentration $c_{B}$ is approximated with a linear gradient between electrode and membrane as twice the electrolyte starting concentration $\mathrm{c}_{0}$ in the anode chamber. $\mathrm{F}$ is Faraday's constant and $\mathrm{z}$ the ionic valence. The transport numbers in the membrane and electrolyte $t_{M}$ and $t_{E}$ describing the ion velocity difference are 0.96 and 0.4 , respectively. For a $1 \mathrm{mM} \mathrm{CuSO}{ }_{4}$ solution, current densities larger than $\mathrm{i}_{\text {lim }}$ $=0.73 \mathrm{~A} \mathrm{~m}^{-2}$ will result in the EC vortex formation close to the membrane

We use micro particle tracking velocimetry ( $\mathrm{PPTV})$, which is an optical technique for measuring fluid flow in millimeter-scale geometries [41] to record the velocity field of EC during our experiments. When using this 
method, tracer particles are illuminated by a high-frequency $532 \mathrm{~nm}$ Nd:YAG laser (DM150, Photonics Industries International Inc.) and recorded by two high-speed cameras (Phantom VEO 710L, Vision Research Inc.) through a stereo microscope (SteREO Discovery.V20, Carl Zeiss Microscopy Deutschland $\mathrm{GmbH}$, Germany) containing a $1 \times$ objective (Plan-Aprochromat 1×, Carl Zeiss Microscopy Deutschland GmbH, Germany), see Fig 3. The cameras record the resulting particle images inside the microscope's focal depth from two different viewing angles. With our setup, the recorded volume at a magnification of $5.12 \times$ and a halfway closed aperture has a size of $4.9 \mathrm{~mm} \times 3.1 \mathrm{~mm} \times 0.8 \mathrm{~mm}$ with $1280 \mathrm{px} \times 800 \mathrm{px}$ resolution in $\mathrm{x}$ - and $\mathrm{y}$-direction. The depth of the recorded volume of $0.8 \mathrm{~mm}$ matches the depth of our electrochemical cell's anode chamber. Therefore, it is possible to reconstruct the full velocity field of EC between membrane and anode.

\subsection{Combining electrochemical experiments and velocity recording}

To conduct simultaneous electrochemical and $\mu \mathrm{PTV}$ experiments, the electrolyte in the anode chamber is seeded with $0.001 \mathrm{wt} \%$ inert, fluorescent polystyrene microspheres (Thermo Scientific, Waltham, MA, USA). These tracer particles have a diameter of $3.2 \mu \mathrm{m}$ and a Zeta potential of $-14.9 \mathrm{mV}$ measured with a Malvern Zetasizer Nano ZS (Malvern Panalytical Ltd), which are comparable to the study of De Valenca et al. [15]. Different to De Valenca et al., the microscope is focused below the membrane in the middle of the anode chamber enabling to record the particle tracks over the whole z-dimension of that chamber. The experiments are conducted without forced flow and electrolyte movement only results from EC.

The chronopotentiometric experiment are conducted at fixed multiples of the limiting current using a potentiostat (Interface 1010E, Gamry, Pennsylvania, USA). For each potential, the time until CP is fully built-up and EC starts to develop can be calculated with Sand's equation [42]:

$$
\tau=\frac{\pi D}{4} \cdot\left(\frac{c_{0} z F}{t_{M}-t_{E}}\right)^{2} \frac{1}{i^{2}}
$$

Here, $c_{0}$ is the electrolyte's initial concentration in the anode chamber. The diffusion coefficient of the electrolyte $\mathrm{D}=0.855 \times 10^{-9} \mathrm{~m}^{2} \mathrm{~s}^{-1}$ is calculated from both individual diffusion coefficients $\mathrm{D}_{\mathrm{Cu}^{2+}}=0.714 \times 10^{-9} \mathrm{~m}^{2} \mathrm{~s}^{-1}$ and $\mathrm{D}_{\mathrm{SO}_{4}{ }^{2-}}=1.065 \times 10^{-9} \mathrm{~m}^{2} \mathrm{~s}^{-1}$ with the following equation [43]: 


$$
D=\frac{\left(z_{+}+z_{-}\right) D_{+} D_{-}}{z_{+} D_{+}+\left|z_{-}\right| D_{-}}
$$

The + and - signs indicate the values for cation and anion, respectively. However, EC takes longer than the calculated transition time $\tau$ to reach a steady-state [24]. Therefore, we analyze the flow well after the transition time in the steady sections of the chronopotentiometry to ensure evaluation of the steady-state.

At $4 \cdot \mathrm{i}_{\text {lim }}$, the formation of the EC vortices takes longer than the electrostatic exclusion of tracer particles from the ESC, effectively preventing recording the EC vortex field. In this experiment, the current density is linearly decreased from $8.1 \cdot \mathrm{i}_{\text {lim }}$ to the desired current density at a rate of $4 \times 10^{-3} \mathrm{~A} \mathrm{~m}^{-2} \mathrm{~s}^{-1}$. Afterward, a steady-state at $4 \cdot \mathrm{i}_{\text {lim }}$ could be measured. This approach ensures the presence of particles close to the membrane due to previous EC mixing of the domain.

When a steady-state in the experiment was reached, the recording of the particle images is started. The flow is recorded for a maximum of $126 \mathrm{~s}$ at a frequency of $20 \mathrm{~Hz}$. The time-resolved recording of the velocity field also enabled the reconstruction of the velocity field during the build-up of EC.

\subsection{Velocity processing}

The recorded particle images are analyzed with the tools available in the software DaVis (version: 10.0.5.47779, LaVision GmbH, Göttingen, Germany). In a preprocessing step, static particle signals are removed by subtracting the time-averaged intensity for each pixel. For the reconstruction of the particle tracks, the Shake-the-Box algorithm is used [44, 45]. However, experiments at current densities below $2 \cdot \mathrm{i}_{\lim }$ cannot be processed. Here, the particles move too slow and the velocities are too small to be detected.

A fine-scale reconstruction is used for transforming the particle tracks to velocities $\left(u_{x}, u_{y}, u_{z}\right)$ on a regular grid. The fine-scale reconstruction is performed with the VIC\# method implemented in the DaVis Software [46, 47].For our experiments, we chose a grid size of ten voxels, which results in reasonable computing times. Therefore, the final resolution of the reconstructed velocity fields is $128 \mathrm{px} \times 80 \mathrm{px} \times 21 \mathrm{px}$ with a voxel size of $38.4 \mu \mathrm{m}$. 
To visualize the vortex structure of $\mathrm{EC}, \mathrm{DaVis}$ offers plotting coherent vortex structures with the $\lambda_{2}$-method [48]. This method computes vortex core lines by evaluating the eigenvalues of the squared and summed symmetric and antisymmetric parts of the gradient velocity tensor. Coherent structures are visualized as isosurfaces at a specific eigenvalue $\lambda_{2}$.

After exporting the velocity field data to MatLab (version: R2019b, The MathWorks Inc.), the mean square velocities is calculated as a spatial and temporal average in the steady-states of the experiments [10]:

$$
\overline{u_{i}^{2}}=\frac{1}{N_{t} \cdot L_{x} \cdot L_{y}} \int u_{i}^{2} \cdot d x \cdot d y \cdot d t
$$

The equation includes the number of time steps $\mathrm{N}_{\mathrm{t}}$ and the mesh points in $\mathrm{x}$ - and $\mathrm{y}$-direction $\mathrm{L}_{\mathrm{x}}$ and $\mathrm{L}_{\mathrm{y}}$.

The fluctuating velocity component is calculated by subtracting the temporal and spatial mean velocity from each velocity data point for evaluation of the temporal and spatial energy spectra. To this data, we apply a Hanning window. The Hanning window or Hann function is a weighting function which is used to reduce the input signal from the middle of the $\mathrm{x}, \mathrm{y}$-domain towards its borders [49]:

$$
w_{i}\left(n_{i}\right)=0.5\left(1-\cos \left(2 \pi \frac{n_{i}}{N_{i}}\right)\right)
$$

Here, $n_{i}$ and $N_{i}$ are the array of data points and the number of data points in $\mathrm{x}$ - or $\mathrm{y}$-direction, respectively. The $2 \mathrm{D}$ window is created with $w_{x, y}\left(n_{x}, n_{y}\right)=w_{x}\left(n_{x}\right) \cdot w_{y}\left(n_{y}\right)$.

For spectral analysis, a Fourier transformation of the fluctuating velocity components over time or space is performed. The velocities, that were 1D Fourier transformed over time $\tilde{u}_{i}$, are then converted to temporal spectral energies and averaged in the $\mathrm{x}, \mathrm{y}$-planes with [10]:

$$
E(z, \omega)=\frac{1}{2 \cdot N_{t} \cdot N_{x} \cdot N_{y}} \int\left|\tilde{u}_{x}\right|^{2}+\left|\tilde{u}_{y}\right|^{2}+\left|\tilde{u}_{z}\right|^{2} d x d y
$$

Here, $N_{t}, N_{x}$, and $N_{y}$ are the number of data points in time, the x-direction, and the y-direction.

For spatial spectral analysis, a 2D Fourier transformation of each fluctuating velocity component in each z-plane for each time step is performed. 
The energy is normalized, and averaged over time:

$$
\begin{aligned}
& E(z, k)= \\
& \frac{1}{2 \cdot N_{t}} \cdot \frac{d^{6}}{(2 \cdot \pi)^{3} \cdot L_{x} \cdot L_{y} \cdot L_{z}} \int\left|\tilde{u}_{x}\right|^{2}+\left|\tilde{u}_{y}\right|^{2}+\left|\tilde{u}_{z}\right|^{2} d t
\end{aligned}
$$

The result is integrated over annuli with a radius of $k=\sqrt{k_{x}^{2}+k_{y}^{2}}$ and a thickness of one in the Fourier-space (see appendix Appendix A). Here, d is the mesh spacing, and $\mathrm{L}_{\mathrm{x}}, \mathrm{L}_{\mathrm{y}}$, and $\mathrm{L}_{\mathrm{z}}$ are the domain sizes in the $\mathrm{x}-, \mathrm{y}-$, and z-directions, respectively. This procedure results in energy graphs over the wavenumber $\mathrm{k}$ which is the spatial frequency in $\mathrm{x}, \mathrm{y}$-direction. During the integration, the mesh is refined by a factor of ten in both directions to generate smoother graphs.

\subsection{Comparability to simulations}

To achive camparability to the 3D DNSs of Druzgalski et al. [12]. We chose process parameters that best match their dimensionless numbers summarized in Tab. 1. The relevant dimensionless parameters concerning this comparison include: i) the applied voltage divided by the thermal voltage $\frac{V}{V_{T}}=\frac{V z e}{k_{B} T}$, ii) the Schmidt number $S c=\frac{\mu}{\rho D}$, iii) the electrohydrodynamic coupling constant $\kappa=\frac{\epsilon}{\mu D} \cdot\left(\frac{k_{B} T}{z e}\right)^{2}$, iv) the dimensionless Debye length $\lambda_{d}=\sqrt{\frac{\epsilon k_{B} T}{2 c_{b}\left(z e L_{z}\right)^{2}}}$, and v) the aspect ratio $a=\frac{d}{L_{z}}$. Here, $k_{B}, T, z$, and $e$ are the Boltzmann's constant, temperature, ionic valence, and the electron charge, respectively. Furthermore, the electrolyte defines the permittivity $\epsilon$, dynamic viscosity $\mu$, diffusion coefficient $\mathrm{D}$, and bulk concentration $\mathrm{c}_{\mathrm{b}}$. Lastly, $d$ and $\mathrm{L}_{\mathrm{z}}$ are the active membrane diameter and the distance between the membrane and electrode, respectively.

Out of these parameters, our main targets were a large channel aspect ratio, a large dimensionless Debye length, and the possibility to conduct experiments at large overlimiting currents, which is proportional to large applied voltages. Advantageous to simulation, our setup allows for dimensions and a Debye length found in practically relevant applications. This difference in Debye length of about three orders of magnitude leads to a smaller extended space charge region in the experiments and, thus, velocity generation closer to the membrane [9]. 
Table 1: Comparison of dimensionless numbers of this study and the reference DNSs [12]. *The applied voltage in our experiments includes the voltage drop at the electrodes, in the membrane, and in the second electrolyte chamber.

\begin{tabular}{lll}
\hline $\begin{array}{l}\text { Dimensionless } \\
\text { number }\end{array}$ & $\begin{array}{l}\text { DNS } \\
{[12]}\end{array}$ & $\begin{array}{l}\text { This } \\
\text { study }\end{array}$ \\
\hline Applied voltage & $120 \frac{V}{V_{T}}$ & $\begin{array}{l}64-362 \\
\frac{V}{V_{T}} * \\
1.17 \times \\
10^{3}\end{array}$ \\
Schmidt number & $1 \times 10^{3}$ & 0.14 \\
Electrohydrodyna- & 0.5 & \\
mic coupling & & \\
constant De- & $1 \times 10^{-3}$ & $6 \times 10^{-6}$ \\
$\begin{array}{l}\text { Dimensionless D Dength } \\
\text { bye length }\end{array}$ & & 10 \\
Aspect ratio & 6.3 & 1.4 \\
i/ilim & 10 & 10.8 \\
\hline \hline
\end{tabular}

\section{Results and Discussion}

Using the electrochemical chip, the movement of tracer particles between the membrane and anode can be followed. Particle tracking velocimetry reveals the $3 \mathrm{D}$ velocity field as a consequence of electroconvection at increasing current densities.

\subsection{Evolution of the velocity field with increasing current density}

First, I-V experiments identify the different current regimes in a current density over potential graph at multiples of the theoretical limiting current density ranging from $0 \cdot i_{\text {lim }}$ to $10.8 \cdot i_{\text {lim }}$, see Fig. 4 a). The plateau is clearly visible between potentials of about $0.4 \mathrm{~V}$ to $0.64 \mathrm{~V}$ matching the calculated theoretical limiting current density of $0.73 \mathrm{~A} \mathrm{~m}^{-2}$ (Eq. 1). At larger potentials, the system enters the overlimiting current region where EC vortices appear. Graphs of the chronopotentiometric experiments are shown in the appendix Appendix B. The data points shown are time-averaged values of the fluctuating signals having their origin in the dynamic nature of the chaotic flow conditions close to the membrane surface. 

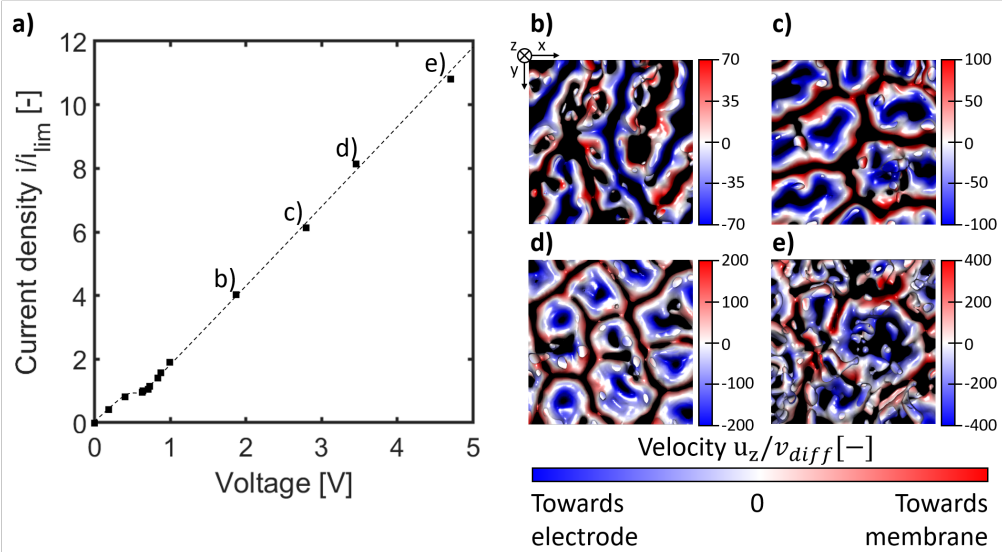

Figure 4: Development of electroconvection in the overlimiting regime. a) The graph shows mean voltages of chronopotentiometry experiments at increasing multiples of the limiting current. The dotted line is drawn for visual guidance. b) - e) Top views on isosurfaces of coherent vortex structures during experiments at b) $4 \cdot i_{\text {lim }}$ (at 90s), c) $6.1 \cdot \mathrm{i}_{\lim }($ at $\left.70 \mathrm{~s}), \mathbf{d}\right) 8.1 \cdot \mathrm{i}_{\lim }($ at $70 \mathrm{~s})$, and e) $10.8 \cdot \mathrm{i}_{\lim }($ at $81 \mathrm{~s})$ in the steady-state. The velocity is colored in magnitude and direction according to the scale bar from blue to red.

We record the velocity fields with our setup during experiments at different magnitudes of the overlimiting current. Fig. 4 b)-e) show snap-shots of the top view on iso-surfaces of coherent vortex structures at $4 \cdot \mathrm{i}_{\text {lim }}, 6.1 \cdot \mathrm{i}_{\text {lim }}$, $8.1 \cdot \mathrm{i}_{\mathrm{lim}}$, and $10.8 \cdot \mathrm{i}_{\mathrm{lim}}$, respectively. With increasing current density, the roll-like structure seen in Fig. 4 b) collapses and vortex rings of oval shape emerge, see Fig. 4 c). With even higher current densities, the vortex rings first become more regular, see Fig. 4 d), before chaotic changing of patterns emerges, see Fig. 4 e). While we show the quantification of such 3D velocity field for the first time, this transition from vortex rolls to vortex rings matches the qualitative observations of Demekhin et al. [11] and Kang et al. [32].

Additionally to the vortex structure, the measurement of velocity vectors enables the evaluation of rotation directions. At $4 \cdot \mathrm{i}_{\text {lim }}$, pairs of counterrotating vortex rolls are the dominant structure. Fig. 4 b) shows that the rolls of a pair connect in the vicinity of other vortex rolls. The velocity between two rolls of a pair is directed towards the membrane. Contrary, the velocity in the centers of vortex rings (Fig. 4 c)-e)) is directed away from 
the membrane. This change in rotation direction indicates that vortex roll pairs do not simply split up in sections and recombine with themselves when transitioning to rings. Two other mechanisms seem more likely: First, vortex roll pairs could split up in sections and recombine but reverse their rotation direction. This change in direction could be induced by the emergence of a smaller vortex ring with a reversed rotation direction in the center of a recombined vortex roll. This smaller ring could increase in size and consume the recombined vortex ring resulting in a ring with a reversed rotation direction. Second, vortex roll pairs could split up at their contact points with other pairs and recombine at the contact point. This observation needs further investigation for a certain explanation.

\subsubsection{Particle tracks of vortex rolls}

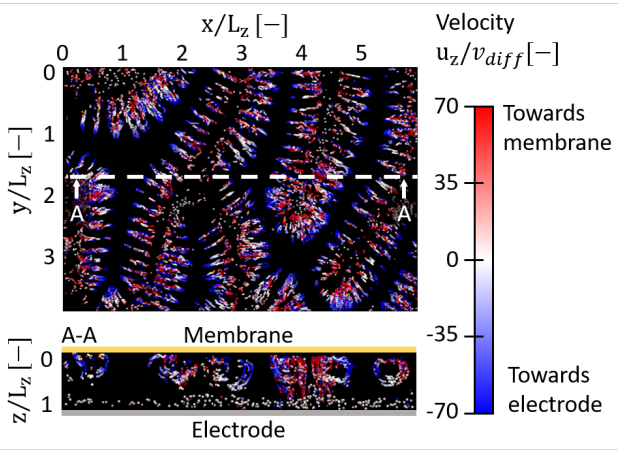

Figure 5: Snapshot of particle tracks for $4 \cdot \mathrm{i}_{\text {lim }}$ at $90 \mathrm{~s}$. Top view of the particle tracks in the reconstructed $3 \mathrm{D}$ volume and a cross-section (A-A) with a track length of 49 time steps $(2.45 \mathrm{~s})$. A-A shows a $2 \mathrm{D}$ cross-section of the $3 \mathrm{D}$ particle field between the electrode and membrane. The velocity is colored in magnitude and direction according to the scale bar from blue to red. The axes are scaled by the channel height $\mathrm{L}_{\mathrm{z}}=800 \mu \mathrm{m}$. Black domains indicate regions where no tracer particles are present due to space charge exclusion as proven by [38]

Fig. 5 shows the $3 \mathrm{D}$ reconstructed particle tracks at $4 \cdot \mathrm{i}_{\text {lim }}$ which is in the region of vortex rolls. The vortex roll pairs, described in the previous section, are visible in the top view as pairs of particle tracks with opposing rotation directions. De Valenca et al. and Davidson et al. already reported that the slight Zeta potential of the tracer particles leads to their exclusion 


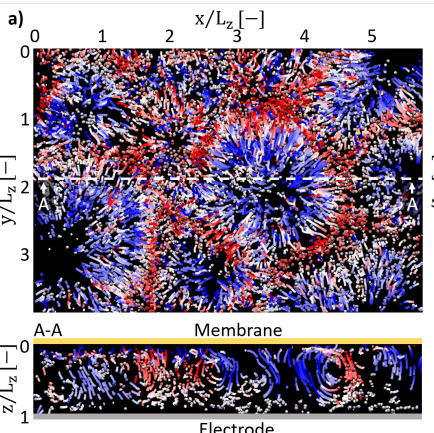

Electrode

from locations with high anion concentration [15, 38]. These locations appear as black areas which are void of particles. Another distinct observation is particles moving on separate orbits along each vortex roll, forming hollow cylindrical structures (Fig. 5). The cross-section A-A shows that the particles are moving on exclusive trajectories. A possible explanation could be the formation of secondary vortices between these separate circles comparable to Taylor flow [50]. These vortices could exclude the particles from the rest of the roll. Another explanation could be the formation of regions of high ion concentration inside the vortex rolls. Such regions would also exclude tracer particles. To identify the validity of these hypotheses, quantitative information on the concentration field would be necessary.

\subsubsection{Veloctity field of chaotic vortex rings}

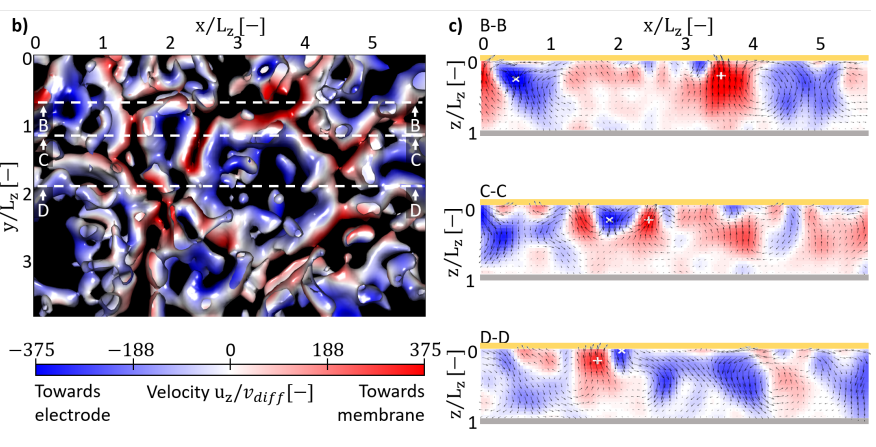

Figure 6: Snapshot of particle tracks and the corresponding velocity correlation for $10.8 \cdot \mathrm{i}_{\text {lim }}$ at $81 \mathrm{~s}$. a) Top view of the particle tracks in the reconstructed $3 \mathrm{D}$ volume with a track length of $1 \mathrm{~s}$. A-A shows a $2 \mathrm{D}$ cross-section of the $3 \mathrm{D}$ particle field between the electrode and membrane. b) Top view of the isosurface of coherent vortex structures in the reconstructed $3 \mathrm{D}$ volume showing a chaotic pattern of vortex rings at $81 \mathrm{~s}$. c) Velocity maps of the cross-section B-B, C-C, and D-D. The white symbols mark the positions of the highest positive (plus) and negative (cross) velocities in each cross-section. The crosssections A-A and D-D show velocity fields calculated from the particle tracks. Videos of the time-resolved particle tracks and vortex structure can be found as supplementary material. The velocity is colored in magnitude and direction according to the scale bar from blue to red.

Fig. 6 shows a snapshot of the steady-state of EC in the regime of chaotic vortex rings at $10.8 \cdot \mathrm{i}_{\text {lim }}$. The top view of reconstructed particle tracks in Fig. 6 a) reveals the appearance of vortex rings with varying diameters. In the cross-section A-A, the particle tracks show circular movement at the 
borders of vortex rolls, for example, at $\mathrm{x} / \mathrm{L}_{\mathrm{z}}=4.5$. These circular tracks strongly resemble structures recorded in the 2D experiments of Valenca et al. [15].

For further investigation, the particle tracks are converted to a 3D velocity field. From this velocity field, coherent vortex structures are extracted and displayed as isosurfaces as shown in Fig. 6 b). The top view of the vortex field structure shows coherent vortex rings as reported by Demekhin et al. [11]. The largest displayed velocities seem to appear in the centers of small vortex rings and at contact points of multiple rings. To confirm this observation, cross-sections of the vortex field through such structures are displayed in Fig. 6 c) B-B, C-C, and D-D. The analysis of the cross-sections prove that the largest absolute velocities in the respective planes appear close to the membrane and between coherent vortex rings. These velocities are up to 4.8 times larger than the average absolute velocities in their respective plane. This velocity distribution can be explained by continuity. The area with velocities towards the electrode is proportionally larger than the area with velocities towards the membrane (Fig. 6 b)) which results in higher velocities for the latter.

These local velocity hot spots might be connected to local current density hot spots at the membrane. At the positions of high velocities towards the membrane, a larger quantity of high-concentrated electrolyte solution is transported from the bulk to the cation-depleted membrane surface. The resulting local jump in cation concentration will, in turn, lead to a local jump in ionic current through the membrane. Thus, the numerically-predicted formation of current density hot spots [12] could be connected to the velocity hot-spot and verified by our experiments.

\subsection{Velocity statistics of electroconvection}

The spatio-temporal 3D velocity information allows to quantify the velocity statistics with increasing current density. This enables to compare the experimental statistics for chaotic vortex rolls to the velocity statistics simulated by Druzgalski et al. from 3D DNSs [12]. 

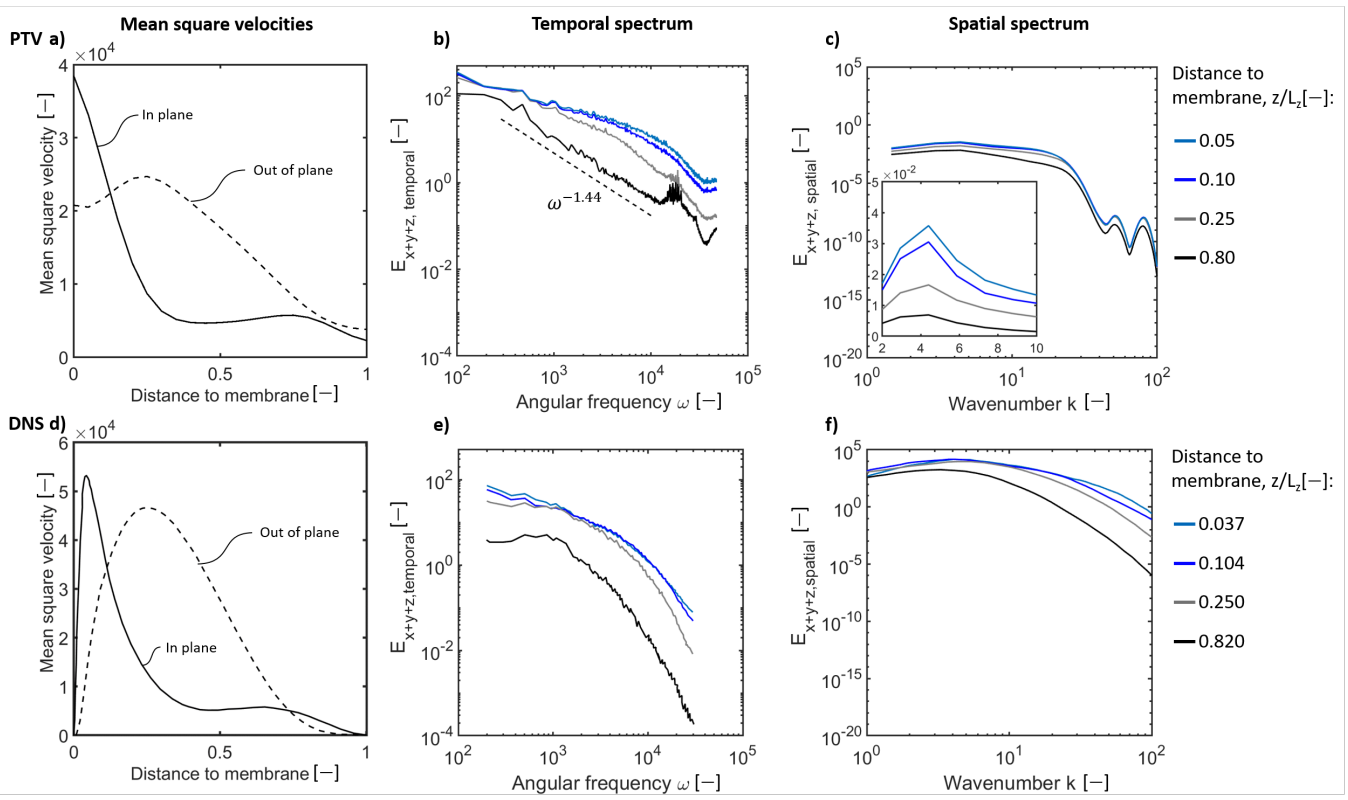

Figure 7: Comparison of mean square velocity, temporal- and spatial energy spectra between 3D $\mu \mathrm{PTV}$ (upper row) and 3D DNSs (lower row) [12]. The graphs present a direct comparison of our $3 \mathrm{D} \mu \mathrm{PTV}$ results at $10.8 \cdot \mathrm{i}_{\mathrm{lim}}$ (1000 time steps, $74.5 \mathrm{~s}$ to $124.5 \mathrm{~s}$ ) a) c) to the 3D DNSs results of Druzgalski et al. d) - f) redrawn from [12] with permission from Physical Review Letters. a) and d) show the mean square of one in-plane and the out-of-plane velocity components over the dimensionless distance to the membrane $z / L_{z}$ with $L_{z}=0.8 \mathrm{~mm}$. b) and e) show temporal energy spectra of the fluctuating velocity components. c) and f) show spatial energy spectra of the fluctuating velocity components. The spectra of $3 \mathrm{D} \mu \mathrm{PTV}$ results b) and c) are plotted for dimensionless distance to the membrane of $0.05,0.10,0.25$, and 0.80 . The linear zoom of the spatial spectrum emphasises the maxima at low wavenumbers. e) and f) are plotted for the normalized distances to the membrane of $0.037,0.104,0.250$, and 0.820 . 


\subsubsection{Comparison of velocity statistics to simulation}

Fig. 7 shows the velocity statistics calculated from our experiment with the statistics extracted from the simulations by Druzgalski et al. [12] for 3D DNSs. The statistical analysis quantifies (a) the mean square velocity and (b) the energy distribution across frequencies.

The mean square velocity for the $3 \mathrm{D} \mu \mathrm{PTV}$ measurements and $3 \mathrm{D}$ simulation [12] are plotted over the normalized distance to the membrane $\mathrm{z} / \mathrm{L}_{\mathrm{z}}$ in Fig. $7 \mathbf{a}$ ) and $\mathbf{b}$ ). The mean squares of the in-plane and out-of-plane velocity components show local maxima at about the same distances to the membrane of $\mathrm{z} / \mathrm{L}_{\mathrm{z}}=0,0.25$, and 0.75 in the experiment and the simulation. A deviation is identified at the $\mathrm{z} / \mathrm{L}_{\mathrm{z}}=0$ and 1 positions that can be attributed to the ideal boundary conditions of the simulations compared to the experiments. The maxima positions originate from the vortex generation close to the membrane leading to the first maximum. The second maximum in the out-of-plane component marks half the vortex height with mostly velocity towards or away from the membrane. The last local maximum in the in-plane velocity is located at the average vortex height where the fluid turns its direction. However, the ratio between the amplitudes of both components differs between our measurements and the DNS.

An explanation for the differences between our measurements and the DNSs of Druzgalski et al. can be the discrepancy in the electric double layer thickness by three orders of magnitude, see Tab. 1. The consequence of this difference is a decreased ESC region and, thus, the appearance of the maximum in-plane velocities closer to the membrane in the experiments [12]. The velocities in z-direction are averaged in segments of $38.4 \mu \mathrm{m}\left(\sim 0.05 \cdot \mathrm{z} / \mathrm{L}_{\mathrm{z}}\right)$ length. This averaging always results in values above zero in the planes next to the membrane and electrode. Compared to DNSs, our experiments also include electrodes, the membrane, and a second electrolyte chamber, which influences the electrochemical cell's resistance and chemistry. This comparison proves that the velocity fields and its statistical mean features originating in the EC can be successfully extracted at practically relevant length and time scales.

The $\mu \mathrm{PTV}$ velocity field data is used to calculate the temporal spectra in planes parallel to the membrane shown in Fig. 7 b). The energy graphs show similar trends in energy levels compared to the numerical results of Druzgalski et al. [12] in Fig 7 e).

Close to the membrane $\left(\mathrm{z} / \mathrm{L}_{\mathrm{z}}=0.05\right.$ and 0.10$)$, the graphs' slope con- 
tinuously decreases from small to large frequencies. At $\mathrm{z} / \mathrm{L}_{\mathrm{z}}=0.25$, a similar energy level is reached in the beginning but shows a more prominent decrease in slope afterwards. The temporal spectrum closest to the electrode notably start at similar energy levels. Different to the DNSs reference, the energy follows a linear decrease that can be fitted by a power-law of $\mathrm{E}_{\mathrm{x}+\mathrm{y}+\mathrm{z} \text {, temporal }} \sim \omega^{-1.44}$ between angular frequencies of $\omega=2.8 \cdot 10^{2}$ and $\omega=1.3 \cdot 10^{4}$. Between frequencies of $\omega=1.3 \cdot 10^{4}$ and $\omega=2.1 \cdot 10^{4}$, fluctuations appear followed by a decrease in energy. All graphs show a change in slope above an angular frequency of $\omega=3.5 \cdot 10^{4}$.

The fluctuations and changes in slope most probably originate from smallscale noise in the experiments and the measurement error of PTV at small scales [51].

The spectra for planes close to the membrane indicate the dissipation of kinetic energy across all frequencies without power-law dependencies, as also reported by Druzgalski et al. for all x,y-planes (Fig. 7 e)) [12]

The power-law relation close to the electrode can be explained by the forces that evoke EC. The body force that generates EC vortices is located close to the membrane [9]. Therefore, vortices will propagate from the membrane towards the electrode. The shape of the spectra also indicates the generation of fluctuations of all time scales at the membrane. The fluctuations with small frequencies, e.g. large vortices, could propagate towards the electrode and dissipate their energy, whereas fluctuations with larger frequencies might not reach the regions close to the electrode.

The spatial energy spectra calculated from our experiment's velocity fields are displayed in Fig $7 \mathbf{c}$ ). All graphs follow the same trend at different energy levels. They show a local maximum at a wavenumber of $\mathrm{k} \sim 4.40$. The slope of the graphs decreases at $\mathrm{k}=22$ with two local maxima above $\mathrm{k}=44$. These local maxima at high frequencies in the spatial $\mu \mathrm{PTV}$ energy spectra can also be linked to small-scale noise and the measurement error of $\mu \mathrm{PTV}$ for small scales [51]. The spatial energy spectra reported by Druzgalski et al. [12] are reproduced in Fig $7 \mathbf{f}$ ). Comparable to our experiment, all graphs show an increase in energy with a maximum at a wavenumber of $\mathrm{k} \sim 4$ [12].

The peak at a wavenumber $\mathrm{k} \sim 4.40$ in the experiment (Fig. 7 c)) corresponds to a length of 1.43 times the electrode-to-membrane distance $\left(\mathrm{L}_{\mathrm{z}}\right)$ carrying the largest energy. This length of $1.43 \cdot \mathrm{L}_{\mathrm{z}}$ matches well with literature and the diameter of the vortex rings in Fig. 6 b).

Due to the time-resolved recording of the velocity field, the evolution of 


\subsection{Development of velocity statistics with increasing current density}
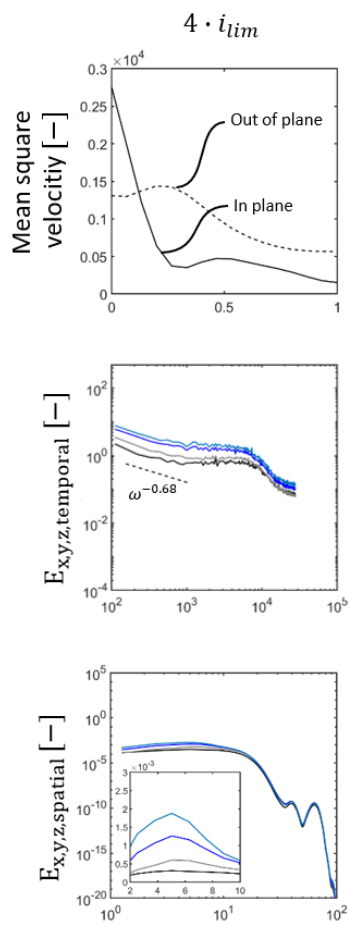
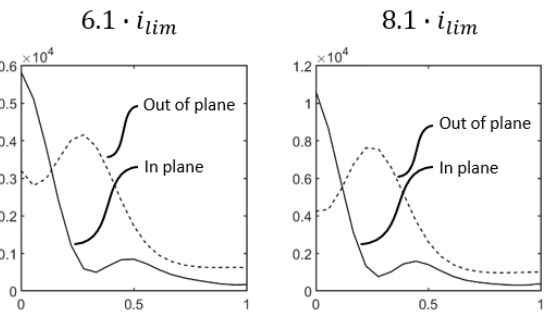

Distance to membrane [-]
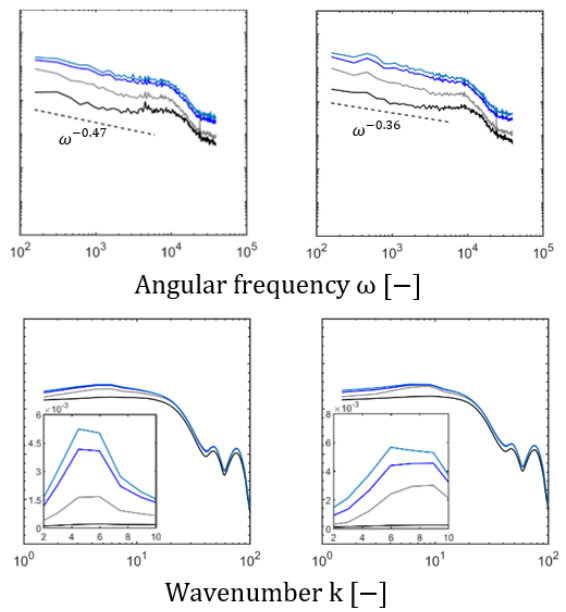

$10.8 \cdot i_{\text {lim }}$
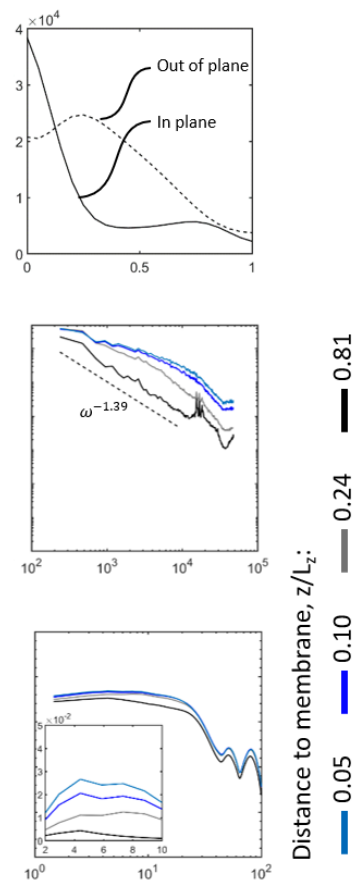

Figure 8: Development of the mean square velocity, temporal and spatial energy spectra in the overlimiting regime for $4 \cdot \mathrm{i}_{\mathrm{lim}}, 6.1 \cdot \mathrm{i}_{\mathrm{lim}}, 8.1 \cdot \mathrm{i}_{\mathrm{lim}}$, and $10.8 \cdot \mathrm{i}_{\mathrm{lim}}$. The graphs represent an average over 1000 time steps $(74.5 \mathrm{~s}$ to $124.5 \mathrm{~s})$. The first row shows the development of the mean square of one in-plane and the out-of-plane velocity components over the plane-to-membrane distance from 3D experiments. The second and third row show the development of the temporal and spatial energy spectra, respectively. Note the changing axes for the graphs showing the mean square velocities and the linear zoom on the maxima of the spatial energy spectra.

Fig. 8 shows the mean square velocity, temporal and spatial energy spectra for the experiments at $4 \cdot \mathrm{i}_{\mathrm{lim}}, 6.1 \cdot \mathrm{i}_{\mathrm{lim}}, 8.1 \cdot \mathrm{i}_{\mathrm{lim}}$, and $10.8 \cdot \mathrm{i}_{\mathrm{lim}}$. All graphs have shapes typical for EC as also seen in Fig. 7. 
The overall mean square velocities increase with an increasing current density, as expected due to the higher energy input. The biggest difference appears between $8.1 \cdot \mathrm{i}_{\lim }$ and $10.8 \cdot \mathrm{i}_{\lim }$. Here, the sharp maximum in the out-of-plane velocity and the local maximum in the in-plane velocity seen at $8.1 \cdot \mathrm{i}_{\text {lim }}$ broaden at $10.8 \cdot \mathrm{i}_{\text {lim }}$. Additionally, the local maximum in the in-plane velocity shifts it's position from $\sim 0.5 \mathrm{~L}_{\mathrm{z}}$ to $\sim 0.75 \mathrm{~L}_{\mathrm{z}}$. This behavior can be explained by the change from the distinct vortex ring pattern into a more chaotic pattern, described in Fig. 4, with vortices that span the whole distance between membrane and electrode as seen in Fig. 6.

The energy spectra in Fig. 8 also reflect the overall higher velocities with increased energies for temporal and spatial fluctuations with increasing current densities. Again, the quality of the results at high frequencies in the temporal as well as the spatial energy spectra suffer from small-scale noise and the measurement error of PTV at small scales [51].

All temporal energy spectra in Fig. 8 can be partly fitted by a powerlaw relation which was also seen in Fig. 7 c). The slope of the fitting first increases from $\omega^{-0.68}$ at $4 \cdot \mathrm{i}_{\lim }$ to $\omega^{-0.36}$ at $8.1 \cdot \mathrm{i}_{\text {lim }}$ and then decreases to $\omega^{-1.39}$ at $10.8 \cdot \mathrm{i}_{\text {lim }}$.

The graphs at $4 \cdot \mathrm{i}_{\text {lim }}$ additionally show a region of constant energy between $\omega=1 \times 10^{3}$ and $\omega=1 \times 10^{4}$ which is less visible at higher current densities. The reason for this steady section could be the absence of information on velocities between the vortex rolls, as seen in Fig. 5. The velocities in these regions potentially fluctuate in small length scales and at high frequencies.

The difference in the temporal energy graphs between $4 \cdot \mathrm{i}_{\text {lim }}$ and $6.1 \cdot \mathrm{i}_{\text {lim }}$ coincides with the structural transition from vortex rolls to vortex rings already seen in Fig. 4. Moreover, the changes between the graphs at $8.1 \cdot \mathrm{i}_{\text {lim }}$ and $10.8 \cdot \mathrm{i}_{\lim }$ match the transition from regular vortex rings to chaotic vortex rings shown in Fig. 4.

In contrast, the shapes of the spatial spectra do not change significantly between current densities. However, the overall energy increases and the maxima emphasised in the linearly scaled inserts change positions from $5.02 \mathrm{k}$ and $4.48 \mathrm{k}$ to $8.95 \mathrm{k}$ and finally to $4.40 \mathrm{k}$. These maxima positions reflect the changes in structure that were seen in Fig. 4. The correlated diameter of the vortex structures first reduces from $6.1 \cdot \mathrm{i}_{\text {lim }}$ to $8.1 \cdot \mathrm{i}_{\text {lim }}$ and then increases again at $10.8 \cdot \mathrm{i}_{\text {lim }}$. The linearly scaled insert at $10.8 \cdot \mathrm{i}_{\text {lim }}$ shows a broader 
energy distribution over multiple wavenumbers compared to the graphs at lower current densities. The absence of a sharp maximum indicates that no single dominant length scale exists but a composition of different length scales. This composition is caused by the chaotic change in vortex structure at this large overlimiting current density with the fluctuating size of vortex rings.

Overall, our experiments confirm that large vortices are the dominant actors during EC [12]. The results additionally indicate that an increasing current density seems to have a more considerable impact on the mean square velocities and temporal spectra than on the spatial spectra.

\section{Conclusion}

We established the first experimental setup for the time-resolved recording of the 3D velocity field during electroconvection (EC) close to a cationexchange membrane. With this setup, Whe EC velocity field was recorded in the steady-state at multiples of the overlimiting current density covering length and time scales found in industrial applications inaccessible by current 3D direct numerical simulations (DNSs).

We visualized coherent vortex structures and revealed the change in vortex structure from vortex rolls to vortex rings along the iV-graph. This change in structure is coherent with the findings reported by Demekhin et al. and Kang et al. [11,32]. Additionally, our method enables evaluating the rotation direction of vortex structures. We found reversed rotation directions between vortex roll pairs and vortex rings, raising the question of the fluid mechanics during the transition from rolls to rings.

The particle tracks in the regime of vortex rings showed the movement of particles on separate orbits. However, possible explanations for this behavior need further physical evidence.

The velocity measurement in the regime of chaotic vortex rings revealed local velocity hot spots between the vortex rings directed towards the membrane. These possibly enable the transport of the cation-rich solution at the electrode towards the membrane. This phenomenon could explain the predicted current density hot spots by 3D DNSs [12].

Further statistical analysis of the velocity field disclosed the nature of energy transfer with a partial power-law behavior in the temporal spectrum. The spatial energy spectrum showed that the length scale carrying the largest 
energy is $1.43 \cdot \mathrm{L}_{\mathrm{z}}$. These findings showed good agreement of general trends with the reported statistics of Druzgalski et al. for 3D DNSs [12]. Differences were found especially for high frequencies.

Lastly, the evolution of the vortex statistics for increasing limiting current densities was analyzed. The results reveal changes in the mean square velocities and the temporal energy spectra during the transition of EC from vortex rolls over vortex rings to chaotic behavior. The spatial energy spectra showed similar trends for all analyzed current densities. These findings indicated a more significant impact of EC's structural change on the temporal spectra than the spatial spectra.

All in all, the presented results provide a first insight of the fundamental physics of EC at practically relevant length and time scales. The experimental technique also builds a foundation to solve the remaining challenges in future detailed 3D studies. Especially the combination of experimental studies, that cover larger scales, and simulations, that more precisely resolve small scales, could lead to significant progress. Furthermore, the methodology permits studying the impact of membrane surface modifications on the hydrodynamics of EC. These modifications aim to manipulate the 3D vortex build-up and reduce the limiting current plateau with increasing success $[52,38,53,54]$ Additionally, knowledge of the velocity statistics enables the development of a RANS-like statistically averaged reduced-order model. Such a model will allow for feasibility analysis and optimization of electrically driven membrane processes in the overlimiting regime at a low computational cost. Full comprehension of EC will push the feasibility of desalination processes beyond the limiting current.

\section{Appendix A. Integration and refinement}

Fig. A.9 displays a graphical representation of the integration and refinement scheme for the spatial spectra also described by Druzgalski et al. [12]. On the left side (Fig. A.9 a)), the two yellow circles with a difference in radius of one form the annuls for integration. The wavenumber $\mathrm{k}$ increases from the center of the k-space in steps of one. The grey highlighted data points show the selection for the non-refined integration. In Fig. A.9 b), a integration scheme with a refinement factor of ten in both directions is displayed. The refined integration results in a smoother graph especially for small wavenumbers by distributing the information in one cell between two 
a)

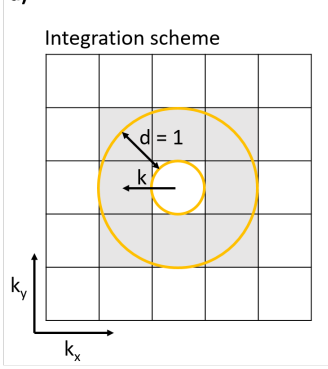

b)

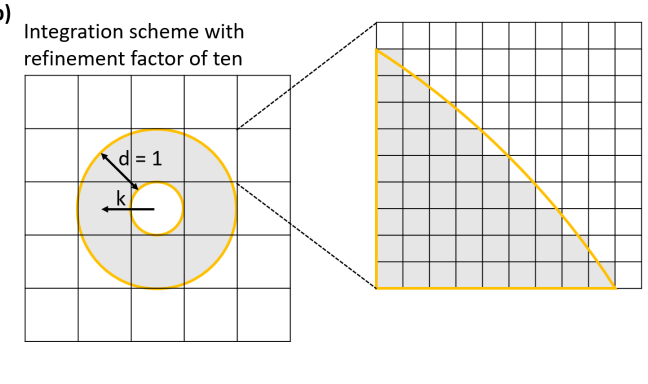

Figure A.9: Graphical representation of the spatial spectra integration and refinement scheme. a) Example of the integration for $\mathrm{k}=1$. b) Example of the refined integration for $\mathrm{k}=1$. The detail on the right shows the selected fraction of the data point in one original pixel

adjacent annuli. The detail on the right shows the selected fraction of the data point.

\section{Appendix B. Chronopotentiometry Graphs}
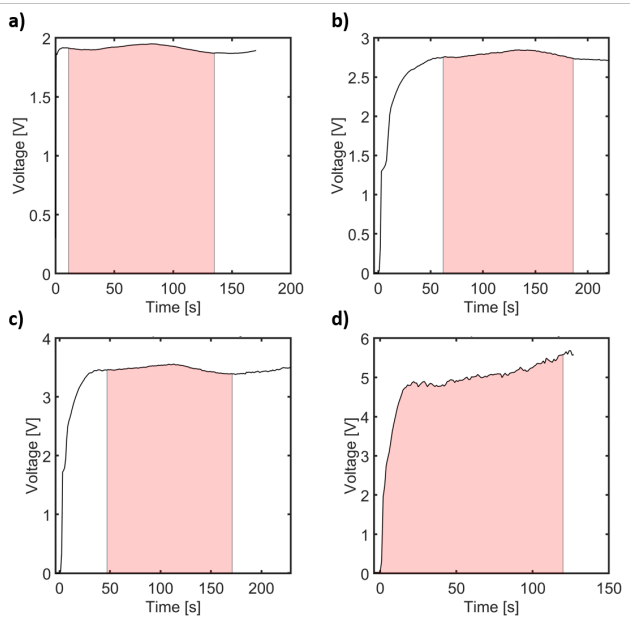

Figure B.10: Chronopotentiometry graphs of the experiments at a) $\left.4 \cdot \mathrm{i}_{\lim }, \mathrm{b}\right) 6.1 \cdot \mathrm{i}_{\lim }$, c) $8.1 \cdot \mathrm{i}_{\mathrm{lim}}$, and d) $10.8 \cdot \mathrm{i}_{\mathrm{lim}}$. The red area displays the recorded sections.

Fig. B.10 shows the chronopotentiometry graphs during the experiments at $4 \cdot \mathrm{i}_{\text {lim }}, 6.1 \cdot \mathrm{i}_{\text {lim }}, 8.1 \cdot \mathrm{i}_{\text {lim }}$, and $10.8 \cdot \mathrm{i}_{\text {lim }}$. The graph in Fig. B.10 a) directly starts at the steady state potential due to the linearly decreased 
current density prior to the experiment as described in the main document's method section.

At current densities of $6.1 \cdot \mathrm{i}_{\text {lim }}$ and $8.1 \cdot \mathrm{i}_{\text {lim }}$, the graphs (Fig. B.10 b) and d)) show a similar trend. After the calculated transition times of $3.94 \mathrm{~s}$ and $2.24 \mathrm{~s}$, the systems resistance increases until EC reaches a steady state.

In the experiment at the highest current density of $10.8 \cdot \mathrm{i}_{\text {lim }}($ Fig. B.10 d)), the potential increases over the course of the measurement. This behavior indicates increasing resistance of the system due to chemical changes or gas bubble formation. Both phenomena could originate from possible water dissociation at the membrane and the electrodes at this large potentials. The resulting $\mathrm{OH}^{-}$ions possibly form solid $\mathrm{CuO}$ or $\mathrm{Cu}(\mathrm{OH})_{2}$ scaling at or inside the membrane [55]. In our experiments, we did not observe any visual changes of neither the membrane nor the electrodes. Excess $\mathrm{H}^{+}$and $\mathrm{OH}^{-}$ would form $\mathrm{H}_{2}$ and $\mathrm{O}_{2}$ gas bubbles at the electrodes. However, gas bubble formation was only observed for experimental times larger than $500 \mathrm{~s}$.

\section{Appendix C. Temporal Development of Chaotic Electroconvec- tion}

Our set up also allows to analyze the development of the EC velocities, vortex structure, and statistics over time. Fig. C.11 and C.12 show this development for the case of $10.8 \cdot \mathrm{i}_{\text {lim }}$ in steps of 20 seconds for the first $120 \mathrm{~s}$ of the experiment.

The development of the coherent vortex structures is depicted in Fig. C.11. The first coherent structures already build up at $20 \mathrm{~s}$ of the experiment appearing as mostly regular vortex rings. The structures grow in diameter over the course of $80 \mathrm{~s}$ while loosing their regular size and shape. Up to this stage, the velocity in most centers of coherent structures is directed towards the electrode. The velocity field becomes more chaotic between $100 \mathrm{~s}$ and $120 \mathrm{~s}$. In this stage, coherent structures appear in a wide range of shapes and sizes with velocities directed both ways.

Fig. C.12 shows the change in one in-plane and the out-of-plane mean square velocities. The distinct velocity profile of EC is not completely build up in the first $20 \mathrm{~s}$. Similar to later stages of EC, the maxima of both velocity components are close to the membrane. However, the out-of-plane velocity is higher than the in-plane component. Both velocity maxima first increase between $20 \mathrm{~s}$ to $40 \mathrm{~s}$ and decrease to a steady state value thereafter. At this point, the profiles are fully developed. 


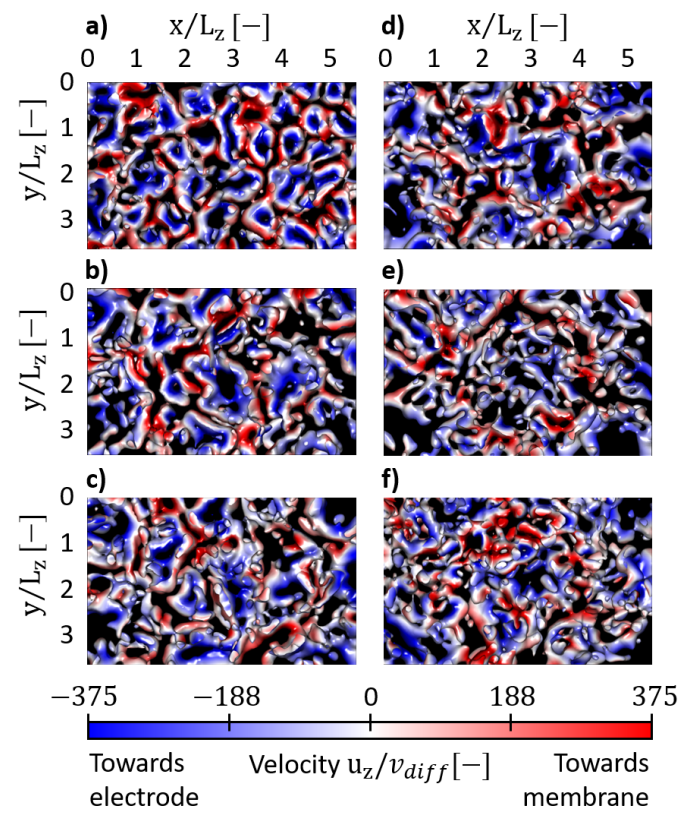

Figure C.11: Temporal development of the EC vortex structure for $10.8 \cdot \mathrm{i}_{\text {lim }}$. The top views of the structure of coherent vortices is depicted at a) $20 \mathrm{~s}$, b) $40 \mathrm{~s}$, c) $60 \mathrm{~s}$, d) $80 \mathrm{~s}$, e) $100 \mathrm{~s}$, and f) $120 \mathrm{~s}$ of the experiment. 


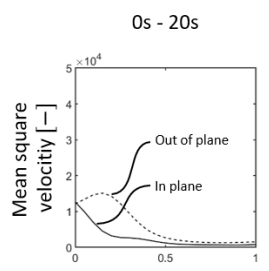

$20 s-40 s$

$40 s-60 s$

$60 s-80 s$

$80 s-100 s$

$100 s-120 s$
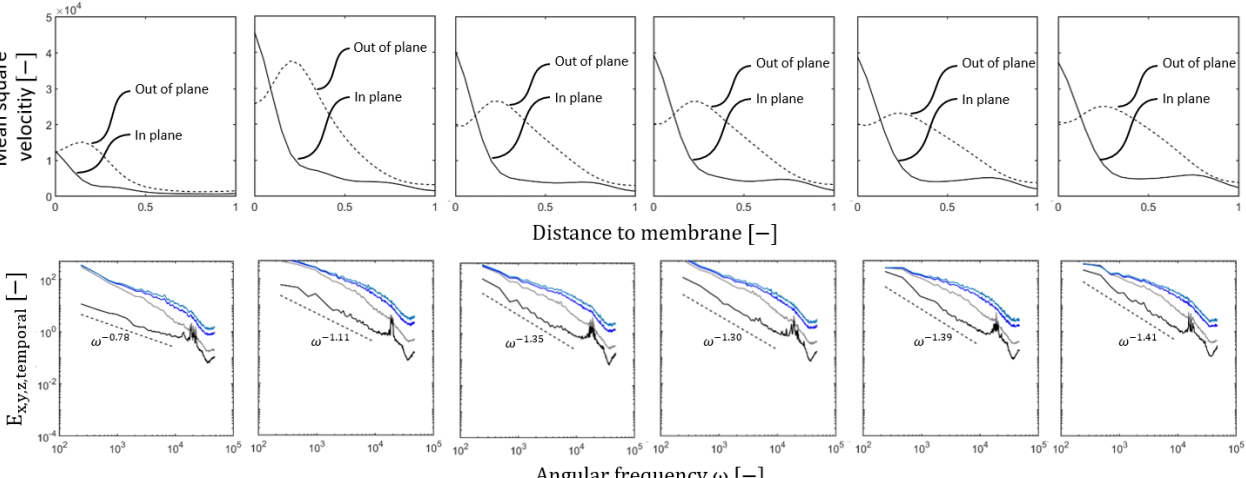

|
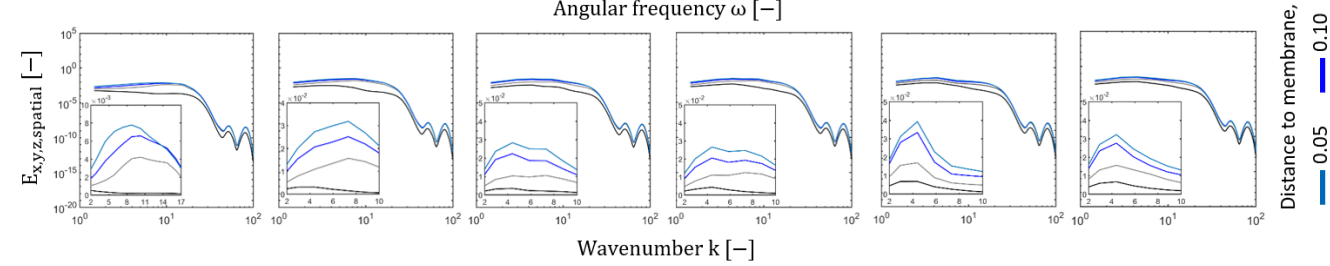

Figure C.12: Temporal development of the mean square velocity, temporal and spatial energy spectra for $10.8 \cdot \mathrm{i}_{\mathrm{lim}}$. The first row shows the development of the mean square of one in-plane and the out-of-plane velocity components over the plane-to-membrane distance from 3D experiments between $0 \mathrm{~s}$ and $120 \mathrm{~s}$. The second and third row show the development of the temporal and spatial energy spectra between $0 \mathrm{~s}$ and $120 \mathrm{~s}$, respectively. Note the changing axes for the graphs showing the linear zoom on the maxima of the spatial energy spectra. 
The above perceptions reflect in the development of the temporal and spatial spectra (Fig. C.12). As explained in the previous section, the local maxima at high frequencies in both the temporal as well as the spatial energy spectra and the following change in slope originate from small-scale noise and the measurement error of PTV for small scales [51]. Only slight changes are visible in the overall shapes of the spectra for both temporal and spatial analysis. However, some distinct observations can be made. First, the slope of the temporal spectrum for the plane closest to the electrode $\left(0.8 \cdot L_{z}\right)$ changes between $0 \mathrm{~s}$ and $40 \mathrm{~s}$ from $\omega^{-0.79}$ to $\omega^{-1.35}$ indicating that the energy reaching the region closer to the electrode dissipates faster when EC is fulls developed. Second, the energy levels in both spectra increase from $0 \mathrm{~s}$ to $20 \mathrm{~s}$ and decrease again after $40 \mathrm{~s}$ as already seen in the mean square velocities. Third, the position of the maxima of the 2D spatial spectra change to smaller wavenumbers for all planes over time. The maxima positions shift from $11.6 k$ to $5.8 k$ between 0 s and 100s as can be seen in the linear zoom of each spectrum. The shift of the maxima correlates to increasing contributions to the energy of vortex structures with larger length scales in the respective planes. The structures with the highest energy share grow from $0.61 \cdot L_{z}$ to $1.43 \cdot L_{z}$ over the course of $80 \mathrm{~s}$. The negligible changes in all graphs confirm that the experiment was in a statistical steady state after about $40 \mathrm{~s}$.

\section{Acknowledgments}

This work received funding from the European Research Council (ERC) under the European Union's Horizon 2020 research and innovation program (grant agreement no. 694946). The DFG also supported this work through the project SFB 985 Functional Microgels and Microgel Systems in project B6. The measurements were conducted with a high-speed stereomicroscope PIV funded by the Major Research Instrumentation Program according to Art. 91b GG in the Research Building NW1481006 "NGP2 - Center for Next Generation Processes and Products". M.W. acknowledges DFG funding through the Gottfried Wilhelm Leibniz Award 2019 (grant ID = WE 4678/12-1) and the support from the Alexander-von-Humboldt foundation. The authors acknowledge F. Roghmans, G. Linz, A. Kalde, J. Lölsberg, A. Cinar, K. Baitalow, and D. Wypysek for their input and the fruitful discussions. 


\section{Author contributions}

Felix Stockmeier: Conceptualization, Methodology, Formal analysis, Investigation, Data Curation, Writing - Original Draft, Visualization, Project administration. Michael Schatz: Formal analysis, Investigation, Visualization. Malte Habermann: Formal analysis, Investigation, Visualization. John Linkhorst: Writing - Review \& Editing, Project administration, Supervision. Ali Mani: Interpretation of the data; Writing - Review \& Editing . Matthias Wessling: Conceptualization, Resources, Project administration, Supervision, Writing - Review \& Editing, Funding acquisition.

\section{Competing interests}

The authors declare that there are no competing interests.

\section{References}

[1] H. Strathmann, Electrodialysis, a mature technology with a multitude of new applications, Desalination 264 (2010) 268-288. doi:10.1016/j.desal.2010.04.069.

[2] J. R. Werber, C. O. Osuji, M. Elimelech, Materials for next-generation desalination and water purification membranes, Nat. Rev. Mater. 1 (2016) 3. doi:10.1038/natrevmats.2016.18.

[3] Y. Gendel, A. K. E. Rommerskirchen, O. David, M. Wessling, Batch mode and continuous desalination of water using flowing carbon deionization (fcdi) technology, Electrochem. Commun. 46 (2014) 152-156. doi:10.1016/j.elecom.2014.06.004.

[4] A. K. E. Rommerskirchen, Y. Gendel, M. Wessling, Single module flowelectrode capacitive deionization for continuous water desalination, Electrochem. Commun. 60 (2015) 34-37. doi:10.1016/j.elecom.2015.07.018.

[5] K. Tang, K. Zhou, Water desalination by flow-electrode capacitive deionization in overlimiting current regimes, Environ. Sci. Technol. 54 (2020) 5853-5863. doi:10.1021/acs.est.9b07591.

[6] M. Park, J. Ryu, W. Wang, J. Cho, Material design and engineering of next-generation flow-battery technologies, Nat. Rev. Mater. 2 (2017) 11533. doi:10.1038/natrevmats.2016.80. 
[7] K. Percin, B. van der Zee, M. Wessling, On the resistances of a slurry electrode vanadium redox flow battery, ChemElectroChem 7 (2020) 2165-2172. doi:10.1002/celc.202000242.

[8] V. V. Nikonenko, A. V. Kovalenko, M. K. Urtenov, N. D. Pismenskaya, J. Han, P. Sistat, G. Pourcelly, Desalination at overlimiting currents: State-of-the-art and perspectives, Desalination 342 (2014) 85-106. doi:10.1016/j.desal.2014.01.008.

[9] A. Mani, K. M. Wang, Electroconvection near electrochemical interfaces: Experiments, modeling, and computation, Annu. Rev. Fluid Mech. 52 (2020) 509-529. doi:10.1146/annurev-fluid-010719-060358.

[10] C. L. Druzgalski, M. B. Andersen, A. Mani, Direct numerical simulation of electroconvective instability and hydrodynamic chaos near an ionselective surface, Phys. Fluids 25 (2013) 110804. doi:10.1063/1.4818995.

[11] E. A. Demekhin, N. V. Nikitin, V. S. Shelistov, Threedimensional coherent structures of electrokinetic instability, Phys. Rev. E: Stat. Nonlinear Soft Matter Phys. 90 (2014) 013031. doi:10.1103/PhysRevE.90.013031.

[12] C. Druzgalski, A. Mani, Statistical analysis of electroconvection near an ion-selective membrane in the highly chaotic regime, Phys. Rev. Fluids 1 (2016). doi:10.1103/PhysRevFluids.1.073601.

[13] S. V. Pham, H. Kwon, B. Kim, J. K. White, G. Lim, J. Han, Helical vortex formation in three-dimensional electrochemical systems with ion-selective membranes, Phys. Rev. E 93 (2016) 033114. doi:10.1103/PhysRevE.93.033114.

[14] Y. Guan, J. Riley, I. Novosselov, Three-dimensional electroconvective vortices in cross flow, Phys. Rev. E 101 (2020) 159. doi:10.1103/PhysRevE.101.033103.

[15] J. C. de Valenca, R. M. Wagterveld, R. G. H. Lammertink, P. A. Tsai, Dynamics of microvortices induced by ion concentration polarization, Phys. Rev. E: Stat. Nonlinear Soft Matter Phys. 92 (2015) 031003. doi:10.1103/PhysRevE.92.031003. 
[16] J. C. de Valenca, Overlimiting current properties at ion exchange membranes, 2017. doi:10.3990/1.9789036543149.

[17] A. Warren, A. Sharma, D. Zhang, G. Li, L. A. Archer, Structure and dynamics of electric-field-driven convective flows at the interface between liquid electrolytes and ion-selective membranes, Langmuir 37 (2021) 5895-5901. doi:10.1021/acs.langmuir.1c00374.

[18] I. Rubinstein, B. Zaltzman, Electro-osmotically induced convection at a permselective membrane, Phys. Rev. E 62 (2000) 2238-2251. doi:10.1103/PhysRevE.62.2238.

[19] E. Korzhova, N. D. Pismenskaya, D. Lopatin, O. Baranov, L. Dammak, V. Nikonenko, Effect of surface hydrophobization on chronopotentiometric behavior of an amx anion-exchange membrane at overlimiting currents, J. Membr. Sci. 500 (2016) 161-170. doi:10.1016/j.memsci.2015.11.018.

[20] K. A. Nebavskaya, V. V. Sarapulova, K. G. Sabbatovskiy, V. D. Sobolev, N. D. Pismenskaya, P. Sistat, M. Cretin, V. V. Nikonenko, Impact of ion exchange membrane surface charge and hydrophobicity on electroconvection at underlimiting and overlimiting currents, J. Membr. Sci. 523 (2017) 36-44. doi:10.1016/j.memsci.2016.09.038.

[21] S. A. Mareev, D. Y. Butylskii, N. D. Pismenskaya, C. Larchet, L. Dammak, V. Nikonenko, Geometric heterogeneity of homogeneous ion-exchange neosepta membranes, J. Membr. Sci. 563 (2018) 768-776. doi:10.1016/j.memsci.2018.06.018.

[22] J. Balster, M. H. Yildirim, D. F. Stamatialis, R. Ibanez, R. G. H. Lammertink, V. Jordan, M. Wessling, Morphology and microtopology of cation-exchange polymers and the origin of the overlimiting current, J. Phys. Chem. B 111 (2007) 2152-2165. doi:10.1021/jp068474t.

[23] M. Wessling, L. G. Morcillo, S. Abdu, Nanometer-thick lateral polyelectrolyte micropatterns induce macrosopic electro-osmotic chaotic fluid instabilities, Sci. Rep. 4 (2015) 4294. doi:10.1038/srep04294.

[24] F. Roghmans, E. Evdochenko, F. Stockmeier, S. Schneider, A. Smailji, R. Tiwari, A. Mikosch, E. Karatay, A. Kühne, A. Walther, 
A. Mani, M. Wessling, 2d patterned ion-exchange membranes induce electroconvection, Adv. Mater. Interfaces 6 (2019) 1801309. doi:10.1002/admi.201801309.

[25] S. M. Rubinstein, G. Manukyan, A. Staicu, I. Rubinstein, B. Zaltzman, R. G. H. Lammertink, F. Mugele, M. Wessling, Direct observation of a nonequilibrium electro-osmotic instability, Phys. Rev. Lett. 101 (2008) 236101. doi:10.1103/PhysRevLett.101.236101.

[26] G. Yossifon, H.-C. Chang, Selection of nonequilibrium overlimiting currents: universal depletion layer formation dynamics and vortex instability, Phys. Rev. Lett. 101 (2008) 254501. doi:10.1103/PhysRevLett.101.254501.

[27] G. Yossifon, P. Mushenheim, Y.-C. Chang, H.-C. Chang, Nonlinear current-voltage characteristics of nanochannels, Phys. Rev. E: Stat. Nonlinear Soft Matter Phys. 79 (2009) 046305. doi:10.1103/PhysRevE.79.046305.

[28] R. Kwak, G. Guan, W. K. Peng, J. Han, Microscale electrodialysis: Concentration profiling and vortex visualization, Desalination 308 (2013) 138-146. doi:10.1016/j.desal.2012.07.017.

[29] R. Kwak, S. V. Pham, K. M. Lim, J. Han, Shear flow of an electrically charged fluid by ion concentration polarization: scaling laws for electroconvective vortices, Phys. Rev. Lett. 110 (2013) 114501. doi:10.1103/PhysRevLett.110.114501.

[30] B. Kim, R. Kwak, H. J. Kwon, S. V. Pham, M. Kim, B. Al-Anzi, G. Lim, J. Han, Purification of high salinity brine by multi-stage ion concentration polarization desalination, Sci. Rep. 6 (2016) 31850. doi:10.1038/srep31850.

[31] T. Bellon, P. Polezhaev, L. Vobecka, M. Svoboda, Z. Slouka, Experimental observation of phenomena developing on ion-exchange systems during current-voltage curve measurement, J. Membr. Sci. 572 (2019) 607-618. doi:10.1016/j.memsci.2018.11.037.

[32] S. Kang, R. Kwak, Pattern formation of three-dimensional electroconvection on a charge selective surface, Phys. Rev. Lett. 124 (2020) 52. doi:10.1103/PhysRevLett.124.154502. 
[33] J. Stodollick, R. Femmer, M. Gloede, T. Melin, M. Wessling, Electrodialysis of itaconic acid: A short-cut model quantifying the electrical resistance in the overlimiting current density region, J. Membr. Sci. 453 (2014) 275-281. doi:10.1016/j.memsci.2013.11.008.

[34] G. Linz, S. B. Rauer, Y. Kuhn, S. Wennemaring, L. Siedler, S. Singh, M. Wessling, 3d-printed bioreactor with integrated impedance spectroscopy for cell barrier monitoring, Adv. Mater. Technol. 6 (2021) 2100009. doi:10.1002/admt.202100009.

[35] E. Karatay, M. B. Andersen, M. Wessling, A. Mani, Coupling between buoyancy forces and electroconvective instability near ion-selective surfaces, Phys. Rev. Lett. 116 (2016) 194501. doi:10.1103/PhysRevLett.116.194501.

[36] J. C. de Valença, A. Kurniawan, R. M. Wagterveld, J. A. Wood, R. G. H. Lammertink, Influence of rayleigh-bénard convection on electrokinetic instability in overlimiting current conditions, Phys. Rev. Fluids 2 (2017). doi:10.1103/PhysRevFluids.2.033701.

[37] P. A. Tsai, Z. A. Daya, S. W. Morris, Aspect-ratio dependence of charge transport in turbulent electroconvection, Phys. Rev. Lett. 92 (2004) 084503. doi:10.1103/PhysRevLett.92.084503.

[38] S. M. Davidson, M. Wessling, A. Mani, On the dynamical regimes of pattern-accelerated electroconvection, Sci. Rep. 6 (2016) 22505. doi:10.1038/srep22505.

[39] Z. Gu, B. Xu, P. Huo, S. M. Rubinstein, M. Z. Bazant, D. Deng, Deionization shock driven by electroconvection in a circular channel, Phys. Rev. Fluids 4 (2019). doi:10.1103/PhysRevFluids.4.113701.

[40] D. Deng, E. V. Dydek, J.-H. Han, S. Schlumpberger, A. Mani, B. Zaltzman, M. Z. Bazant, Overlimiting current and shock electrodialysis in porous media, Langmuir 29 (2013) 16167-16177. doi:10.1021/la4040547.

[41] J. G. Santiago, S. T. Wereley, C. D. Meinhart, D. J. Beebe, R. J. Adrian, A particle image velocimetry system for microfluidics, Exp. Fluids 25 (1998) 316-319. doi:10.1007/s003480050235. 
[42] J. Krol, Chronopotentiometry and overlimiting ion transport through monopolar ion exchange membranes, J. Membr. Sci. 162 (1999) 155-164. doi:10.1016/S0376-7388(99)00134-9.

[43] D. R. Lide (Ed.), CRC handbook of chemistry and physics, 2003-2004: A ready-reference book of chemical and physical data, 84th ed. ed., CRC Press, Boca Raton, Fla., 2003.

[44] D. Schanz, A. Schröder, S. Gesemann, D. Michaelis, B. Wieneke, 'shake the box': A highly efficient and accurate tomographic particle tracking velocimetry (tomo-ptv) method using prediction of particle positions, in: PIV13; 10th International Symposium on Particle Image Velocimetry, 2013. URL: http://resolver.tudelft.nl/uuid:b5eb6d27-bfb14c25-bc79-637df9c76694.

[45] A. Schröder, D. Schanz, D. Michaelis, C. Cierpka, S. Scharnowski, C. J. Kähler, Advances of piv and 4d-ptv "shake-the-box" for turbulent flow analysis - the flow over periodic hills, Flow Turbul. Combust. 95 (2015) 193-209. doi:10.1007/s10494-015-9616-2.

[46] Y. J. Jeon, J. Schneiders, M. Müller, D. Michaelis, B. Wieneke, 4d flow field reconstruction from particle tracks by vic+ with additional constraints and multigrid approximation, in: Proceedings 18th International Symposium on Flow Visualization, 2018. doi:10.3929/ETHZ-B000279199 .

[47] J. F. G. Schneiders, F. Scarano, Dense velocity reconstruction from tomographic ptv with material derivatives, Exp. Fluids 57 (2016) 1. doi:10.1007/s00348-016-2225-6.

[48] J. Jeong, F. Hussain, On the identification of a vortex, J. Fluid Mech. 285 (1995) 69. doi:10.1017/S0022112095000462.

[49] O. M. Essenwanger, Elements of statistical analysis, volume / ed. by O. M. Essenwanger ; 1,B of World survey of climatology General climatology, Elsevier, Amsterdam, 1986.

[50] A. G. Fane, Techniques to enhance performance of membrane processes, in: Handbook of membrane separations, 2009, pp. 193-232. 
[51] S. Herpin, C. Y. Wong, M. Stanislas, J. Soria, Stereoscopic piv measurements of a turbulent boundary layer with a large spatial dynamic range, Exp. Fluids 45 (2008) 745-763. doi:10.1007/s00348-008-0533-1.

[52] J. Balster, M. H. Yildirim, D. F. Stamatialis, R. Ibanez, R. G. H. Lammertink, V. Jordan, M. Wessling, Morphology and microtopology of cation-exchange polymers and the origin of the overlimiting current, J. Phys. Chem. B 111 (2007) 2152-2165. doi:10.1021/jp068474t.

[53] J. C. de Valenca, M. Jõgi, R. M. Wagterveld, E. Karatay, J. A. Wood, R. G. H. Lammertink, Confined electroconvective vortices at structured ion exchange membranes, Langmuir 34 (2018) 2455-2463. doi:10.1021/acs.langmuir.7b04135.

[54] A. M. Benneker, B. Gumuscu, E. G. H. Derckx, R. G. H. Lammertink, J. C. T. Eijkel, J. A. Wood, Enhanced ion transport using geometrically structured charge selective interfaces, Lab Chip 18 (2018) 1652-1660. doi:10.1039/c7lc01220a.

[55] J.-H. Chang, A. V. Ellis, C.-H. Tung, W.-C. Huang, Copper cation transport and scaling of ionic exchange membranes using electrodialysis under electroconvection conditions, J. Membr. Sci. 361 (2010) 56-62. doi:10.1016/j.memsci.2010.06.012. 